\title{
ACTAS DE TALLER: HACIA UN PLAN PARA LA CONSERVACIÓN DEL HUEMUL HIPPOCAMELUS BISULCUS (MOLINA, 1782) EN LA ZONA AUSTRAL DE CHILE. (11-12 DE NOVIEMBRE 2010, PUNTA ARENAS)
}

\section{PROCEEDINGS OF WORKSHOP: TOWARDS AN ACTION PLAN FOR THE CONSERVATION OF HUEMUL HIPPOCAMELUS BISULCUS (MOLINA, 1782) IN SOUTHERNMOST CHILE.} (11 \&12 NOVEMBER, 2010, PUNTA ARENAS)

Claudia Silva ${ }^{1}$, Fiorella Repetto ${ }^{1}$, Daniela Droguett ${ }^{1}$, Claudio Moraga ${ }^{1} \&$ Alejandro Vila ${ }^{1}$

(Editores Actas del Taller)

\begin{abstract}
The huemul is the most threatened cervid of the Neotropical Region. It is estimated that its historic distribution range in Chile has shrunk in over 50\%, while its global population size would not exceed 2500 individuals. Taking into consideration this critical situation, the Workshop "Towards an Action Plan for the Conservation of Huemul in the Austral Zone" was held between November 11th and 12th 2010 in Punta Arenas, Chile, in a joint initiative of the Wildlife Conservation Society, the National Forestry Corporation and the CEQUA Foundation. This Workshop is in line with the implementation process of the National Plan for the Conservation of Huemul in Chile. The objective of this workshop was to initiate the drawing up of an Action Plan that takes into account that special features of the Austral Zone for the conservation of the species.

The Workshop started with oral presentations aiming to update the participants on the current state of the huemul in the target area. Subsequently, a participative approach was used to determine the direct and indirect threats to the species, as well as to prioritize the former. The five direct threats identified were, in order of importance: 1) competition with livestock, 2) habitat loss and fragmentation, 3) diseases, 4) predation and harassment by dogs, and 5) poaching. In a second stage, the participants were divided into four groups: policy and legislation, management and conservation, research and monitoring, and education and communications. Each group made a list of potential interventions related to the identified threats that were considered to be within the scope of the group.
\end{abstract}

1 Wildlife Conservation Society - Programa Chileno -Avda. Gral. Bustamante 144 Of. 42, Providencia, Santiago / Balmaceda 586, Punta Arenas. 
The production of a prioritized list of direct threats and of potential interventions is a significant step forward towards the drawing up of the Action Plan for the Austral Zone. While the proposed interventions vary according to the scope of each group, some recurrently mentioned measures were: the creation of a geographic information system on the current distribution of huemul allowing to contrast it with the spatial distribution of the threats; the coordination among agencies for the management of threats both in and outside protected areas; and the raising of awareness on the situation of huemul among stakeholders of the areas related to identified threats.

During the Workshop, the need to establish measurable objectives and eventually a goal specific to the Austral Zone was apparent. In addition, the discussion about the funding mechanisms for interventions and the drawing up of a monitoring and assessment program remain to be resolved, as does the definition of the agencies/people in charge of implementing the interventions.

Key words: Huemul deer, national plan of conservation, action plan, austral zone, Chile

\section{RESUMEN}

El huemul es el cérvido más amenazado de la Región Neotropical. Se estima que su distribución histórica en Chile ha sufrido una reducción superior al 50\%, mientras que la población mundial no superaría los 2.500 individuos. Considerando esta crítica situación, los días 11 y 12 de Noviembre de 2010 se realizó, en Punta Arenas, el Taller "Hacia un Plan de Acción para la Conservación del Huemul en la Zona Austral", iniciativa conjunta de Wildlife Conservation Society, la Corporación Nacional Forestal y la Fundación CEQUA. Este Taller se enmarca dentro del proceso de implementación del Plan Nacional de Conservación del Huemul en Chile. Su objetivo fue dar inicio a la elaboración de un Plan de Acción que considere las particularidades de la Zona Austral para la conservación de la especie.

El Taller se inició con una serie de exposiciones dirigidas a actualizar a los participantes sobre el estado del huemul en el área blanco. Posteriormente, se usó un enfoque participativo para establecer las amenazas directas e indirectas que afectan a la especie, como así también la priorización de las primeras. Las cinco amenazas directas identificadas fueron, en orden de prioridad: 1) competencia con ganado, 2) pérdida y fragmentación del hábitat, 3) enfermedades, 4) depredación y acoso por perros, y 5) caza furtiva. En una segunda etapa, los participantes fueron divididos en cuatro grupos temáticos: políticas y legislación, manejo y conservación, investigación y monitoreo, y educación y difusión. Cada grupo temático definió un listado de intervenciones potenciales asociadas con las amenazas identificadas que eran relevantes a su ámbito de acción.

La elaboración de la lista priorizada de amenazas directas y de posibles intervenciones ha sido un avance importante para la elaboración del Plan de Acción de la Zona Austral. Si bien las intervenciones propuestas varían según el ámbito de acción de los grupos de trabajo, algunas de las medidas necesarias que se mencionaron recurrentemente fueron: la creación de un sistema de información geográfica sobre la distribución actual del huemul que pueda ser contrastado con la distribución espacial de las amenazas; la coordinación entre instituciones para el manejo de amenazas tanto dentro como fuera de las Áreas Silvestre Protegidas; y la concientización sobre la problemática del huemul a nivel de tomadores de decisiones en áreas relacionadas con las amenazas identificadas.

Dentro de los resultados del Taller se hizo evidente la necesidad de definir objetivos y eventualmente una meta que sean cuantificables y específicos para la Zona Austral. Asimismo, quedó pendiente la discusión sobre mecanismos de financiamiento para la implementación de las intervenciones y el desarrollo de un programa de monitoreo y evaluación, además de la identificación de las personas/instituciones encargadas de implementar las acciones propuestas.

Palabras clave: Huemul, plan nacional de conservación, plan de acción, zona austral, Chile 
El huemul (Hippocamelus bisulcus Molina, 1782) es el cérvido más amenazado de la Región Neotropical. Este ciervo endémico de Chile y la Argentina es el único miembro de esta familia que está clasificado como "En Peligro" en la Lista Roja de Especies Amenazadas de la Unión Internacional para la Conservación de la Naturaleza (UICN) en toda América, dado su pequeño tamaño poblacional y su rango de distribución restringido y fragmentado (Jiménez et al. 2008). Se estima que su distribución histórica en Chile habría sufrido una reducción superior al 50\% (Drouilly 1983), mientras que la población actual no superaría los 2.500 individuos a nivel mundial (Jiménez et al. 2008) y solo representaría un $1 \%$ de su tamaño original (Redford \& Eisenberg 1992). Las principales causas que habrían llevado al huemul a esta situación son la caza y la ocupación y transformación de su hábitat por el hombre. En el presente, sin embargo, la discontinuidad del hábitat ha resultado, en muchos casos, en que la especie persista en pequeñas sub-poblaciones que son vulnerables frente a cualquier perturbación (Caughley 1994, Jiménez et al. 2008).

Considerando esta crítica situación, la Corporación Nacional Forestal (CONAF), en estrecha colaboración con el Comité Nacional Pro Defensa de la Fauna y Flora (CODEFF), publicó en el año 2001 el primer Plan Nacional para la Conservación del Huemul del Sur en Chile (CONAF \& CODEFF 2001). Posteriormente, en 2007 se realizó un Taller con el fin de actualizar este Plan, incorporando nueva información y la visión de un mayor grupo de actores, además de definir, dentro de lo posible, metas y actividades cuantificables que permitieran evaluar su avance y efectividad. Finalmente, en 2009 fue publicado el Plan Nacional de Conservación del Huemul (Hippocamelus bisulcus Molina, 1782) en Chile: 2008-2012 (CONAF et al. 2009).

El Plan Nacional está coordinado por un Comité Ejecutivo Nacional y espera concretar su implementación, a escala local, a través de tres grupos de trabajo zonales, uno de los cuales es el Grupo de la Zona Austral (CONAF et al. 2009; Fig. 1 ). Se presume que las poblaciones más numerosas, interconectadas y mejor conservadas de la especie se encuentran en esta zona (López et al. 1998, Vila et al. 2006, CONAF et al. 2009, Vila et al. 2010). Si bien esta situación representa una oportunidad para la conservación de la especie, es importante considerar que, a semejanza con el resto de su rango de distribución, las poblaciones en la Zona Austral también enfrentan diversas e importantes amenazas. Por lo tanto, la tarea prioritaria para este Grupo de trabajo es elaborar un Plan de Acción que considere las particularidades zonales para implementar de manera efectiva el Plan Nacional en la Zona Austral de Chile.

En este contexto, los días 11 y 12 de Noviembre de 2010, se llevó a cabo en la ciudad de Punta Arenas el Taller 'Hacia un Plan de Acción para la Conservación del Huemul en la Zona Austral', iniciativa conjunta de Wildlife Conservation Society (WCS), la Fundación CEQUA y la Corporación Nacional Forestal (CONAF). El objetivo del Taller fue dar inicio, de manera participativa, a la elaboración del Plan de Acción para la Conservación del Huemul en la Zona Austral. El comité Organizador estuvo integrado por Juan Carlos Aravena (CEQUA); Daniela Droguett, Claudio A. Moraga, Fiorella Repetto, Alejandro Vila (WCS); Alejandra Silva, Carla Hernández y Mauricio Ruiz (CONAF).

\section{ENFOQUE METODOLÓGICO}

Se realizó un taller de expertos como un primer paso para la elaboración del Plan de Acción, con un trabajo participativo para: a) introducir esta iniciativa de conservación entre actores clave; b) obtener una panorámica más completa del contexto de implementación, al incluir diversas visiones; c) generar un espacio de interacción para construir confianza entre actores y d) obtener conclusiones validadas mediante el consenso, aumentando así la probabilidad de éxito de las medidas a implementar (WCS 2004).

Se extendió una invitación a 60 profesionales y especialistas pertenecientes a distintas instituciones públicas o privadas relacionadas con la conservación de la especie, su hábitat y/o el manejo de recursos naturales. Como catorce de ellos se excusaron de asistir, el Taller contó finalmente con la participación de 46 asistentes (Anexo 1).

El Taller tomó como base el desarrollo de un modelo conceptual para establecer una meta y los objetivos de conservación, amenazas directas e indirectas que afectan el cumplimiento de los mismos $y$, finalmente, las intervenciones que se deberían tomar para mitigar o eliminar estas amenazas (Fig. 


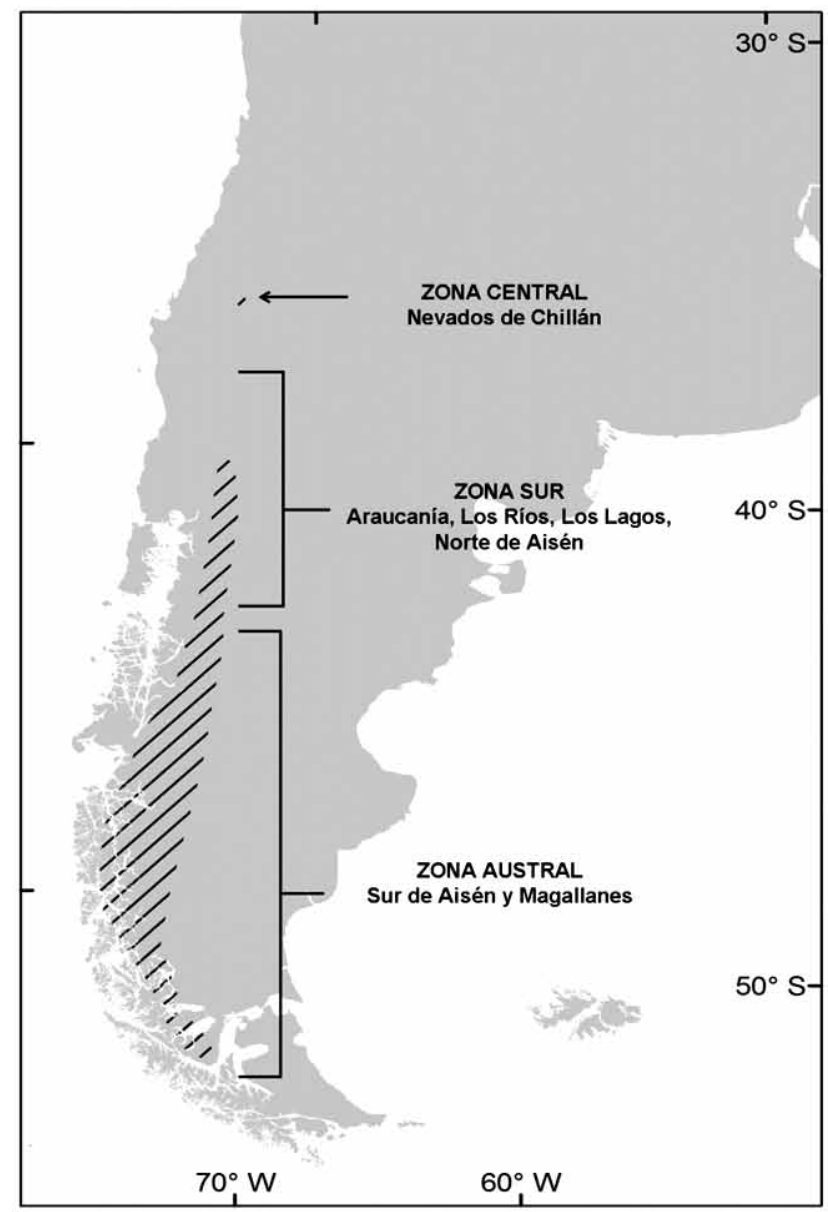

Fig. 1. Zonas geográficas identificadas por el Plan Nacional que, debido a sus características particulares, requieren de medidas específicas de conservación. Las líneas diagonales indican la distribución actual potencial del huemul en Chile y la Argentina.

2). A continuación se definen los conceptos utilizados en este enfoque:

Modelo conceptual: Es un esquema que expresa de manera clara y simplificada la meta y los objetivos de conservación, las amenazas directas $e$ indirectas y las intervenciones necesarias para alcanzar la meta de conservación.

Meta: Es una idea general y resumida sobre la situación que intentamos influenciar a través de las actividades del Plan. Debe ser general y breve, pero a la vez mensurable.

Objetivos: Son enunciados específicos que detallan los logros o resultados deseados por el Plan. Deben ser mensurables, limitados y prácticos.

Amenazas directas: Son factores que ocasionan la destrucción física del objeto de conservación. Estas amenazas suelen manifestarse como pérdida de hábitat, eliminación o sobre-explotación de especies, contaminación e introducción de especies exóticas (WCS 2004, 2006), las cuales se corresponden con los principales agentes que causan pérdida de biodiversidad (Diamond 1984).

Amenazas indirectas: Son factores que ocasionan o influencian a las amenazas directas (WCS 2004, 2006). Son el resultado de la interacción entre los usuarios de un recurso, los administradores del mismo y las políticas que regulan esta interacción. Intervenciones: Son acciones o tareas específicas a desarrollar en el marco del Plan. Están diseñadas para alcanzar los objetivos propuestos. Deben ser factibles considerando los recursos disponibles.

La dinámica de trabajo del Taller se dividió en cuatro sesiones. La Sesión 1 consistió en una serie de exposiciones sobre el estado actual del huemul 


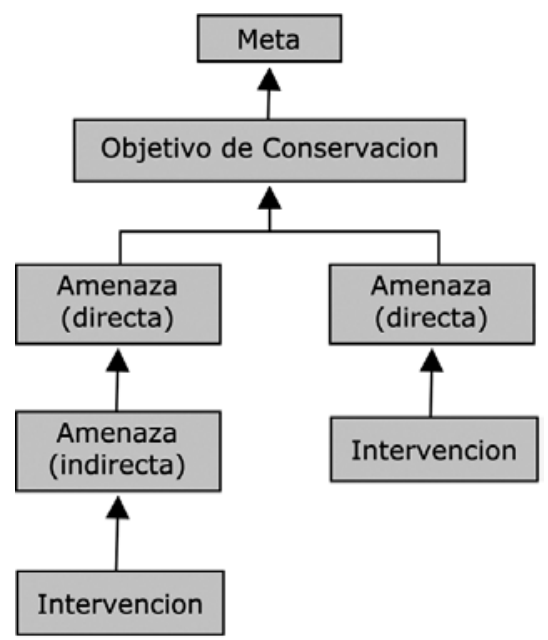

Fig. 2. Modelo conceptual hipotético sobre el cual se basó el trabajo del Taller.

y las medidas de conservación y manejo que se han tomado en la Zona (Anexos 2 y 3). El objetivo de esta sesión fue actualizar y concientizar a los participantes sobre la situación del huemul, así como comprometerlos con la implementación del Plan de Acción. En la Sesión 2 se presentó la metodología de trabajo. Se revisó tanto la meta como los objetivos del Plan Nacional. En el caso de las amenazas, se buscó identificar aquellas relevantes para la Zona Austral. Las sugerencias de los participantes fueron agrupadas y discutidas para luego elaborar listados consensuados de las mismas. Las amenazas directas fueron ordenadas de mayor a menor grado de prioridad, en base a los resultados de un ejercicio de votación realizado con los participantes. Cada uno de ellos votó, según su percepción de experto, por las tres amenazas directas que consideraba más importantes (WCS 2006).

Durante la Sesión 3 se buscó establecer la relación existente entre amenazas directas e indirectas y definir las acciones y medidas necesarias para mitigarlas o abatirlas. Según su afiliación y experiencia, los participantes conformaron uno de los siguientes grupos de trabajo: 1) políticas y legislación; 2) manejo y conservación de la especie y su hábitat; 3) investigación y monitoreo y 4) educación y comunicaciones. Cada grupo contó con dos facilitadores que estuvieron a cargo de dirigir el proceso de discusión. Cada grupo revisó la lista ordenada de amenazas directas e indirectas, excluyendo aquellas que se consideraban fuera del alcance de su área acción.

\section{DESARROLLO DEL TALLER}

\section{Meta y objetivos del Plan de Acción}

Se discutió la meta y los objetivos actuales del Plan Nacional. Se reconocieron algunas falencias en cuanto a la definición de los objetivos. Al no haber sido enunciados en forma cuantificable y considerando un marco de tiempo para alcanzarlos, sería difícil evaluarlos. Se consideró que los enunciados eran muy generales y poco orientados. Si bien la discusión fue breve, se consideró apropiado que a futuro se definan objetivos que sean cuantificables y específicos para las poblaciones de la Zona Austral.

\section{Amenazas directas e indirectas}

Se compiló en plenario una lista de 11 amenazas directas y 11 indirectas (Tabla 1). En

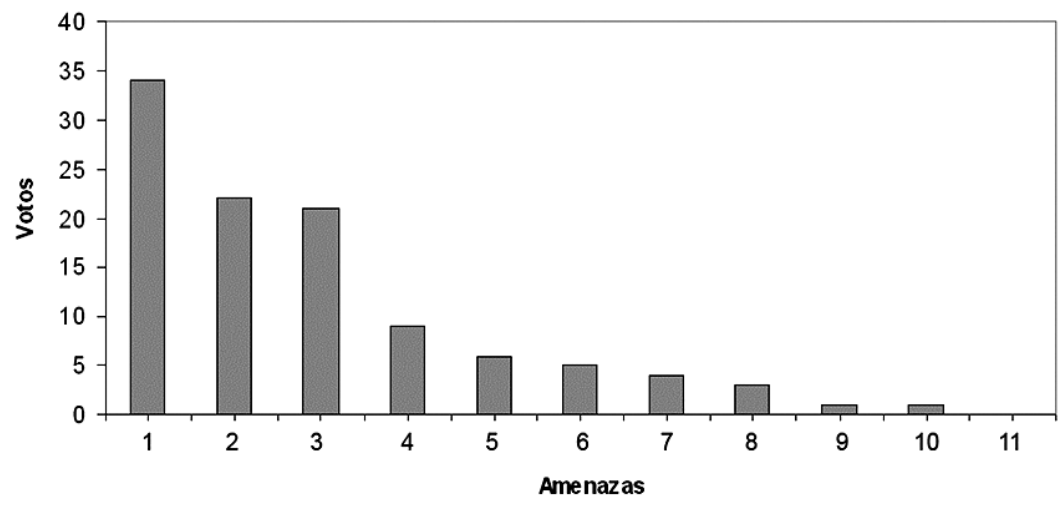

Fig. 3. Resultado de la votación para priorizar amenazas directas. Cada amenaza está representada por su ID (ver Tabla 1). 
Tabla 1. Listado de amenazas directas e indirectas identificadas en el Taller. Las amenazas directas se presentan en orden de mayor a menor importancia según la votación realizada. ID= identificador.

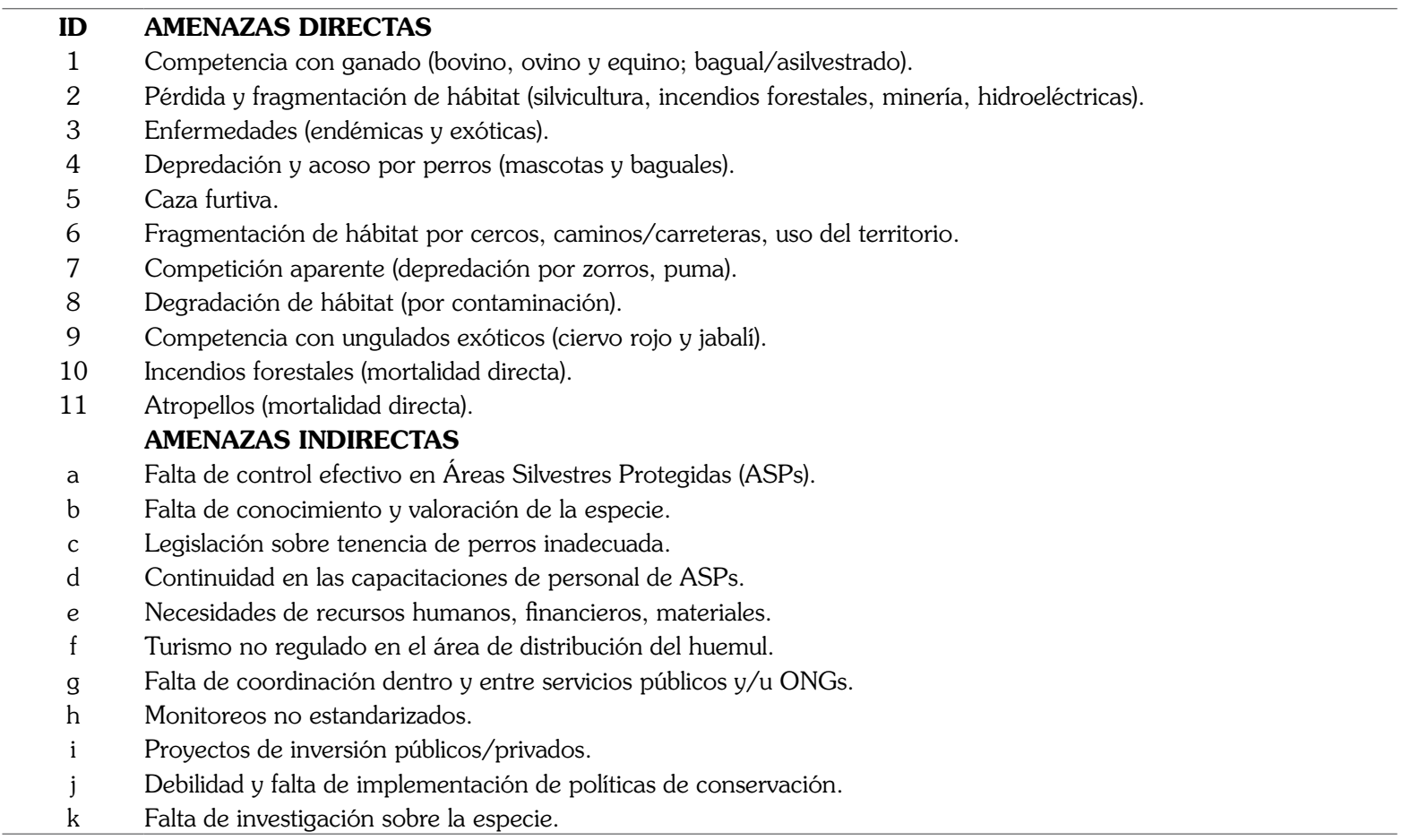

base a esta lista se realizó una votación, la cual arrojó el orden de prioridad presentado en la Fig. 3 . Para focalizar el ejercicio siguiente de identificación de intervenciones se decidió restringir la discusión a las primeras cinco amenazas de la lista, las cuales concentraron el $87 \%$ de los votos de los participantes.
Análisis de Amenazas y Propuesta de Intervenciones para Políticas y Legislación:

Este grupo estuvo conformado por J.L. Cabello, R. Contreras, D. Droguett, R. López, A. Meidinger, G. Stipicic, G. Santana, J. C. Tonko y C. Venegas. El mismo identificó las siguientes relaciones entre amenazas:

\begin{tabular}{|c|c|c|c|c|c|}
\hline Matriz de relación entre amenazas directas e indirectas & 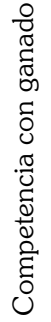 & 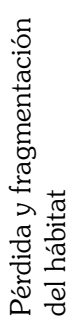 & 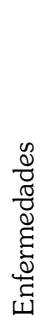 & 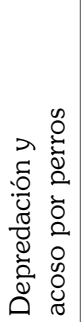 & $\begin{array}{l}\underset{Z}{Z} \\
\underset{J}{J} \\
\mathbb{N} \\
\widetilde{J}\end{array}$ \\
\hline \multicolumn{6}{|l|}{ Falta de control efectivo en ASPs } \\
\hline \multicolumn{6}{|l|}{ Falta de conocimiento y valoración de la especie } \\
\hline \multicolumn{6}{|l|}{ Legislación sobre tenencia de perros inadecuada } \\
\hline \multicolumn{6}{|l|}{ Necesidad de recursos humanos, financieros y materiales } \\
\hline \multicolumn{6}{|l|}{ Turismo no regulado en la distribución del huemul } \\
\hline \multicolumn{6}{|l|}{ Falta de coordinación dentro y entre servicios públicos y/u ONGs } \\
\hline \multicolumn{6}{|l|}{ Proyectos de inversión públicos/privados } \\
\hline \multicolumn{6}{|l|}{ Debilidad y falta de implementación de políticas de conservación } \\
\hline Falta de investigación de la especie & & & & & \\
\hline
\end{tabular}


A continuación se enumeran las acciones propuestas por este grupo:

1.- Competencia con ganado.

1.1.- Erradicar ganado doméstico o bagual de las áreas protegidas con presencia de huemul (se sugiere a CONAF como organismo encargado).

1.2.- Erradicar el ganado bagual de las zonas fuera de áreas protegidas con presencia de huemul (se sugiere a SAG como organismo encargado).

1.3.- Difundir buenas prácticas de manejo ganadero en hábitat con presencia de huemul.

2.- Pérdida y fragmentación de hábitat.

2.1.- Integrar al Ministerio de Bienes Nacionales (MNB) al Plan de Acción, para que incorpore al huemul dentro de sus prioridades al evaluar opciones de uso y manejo de sitios fiscales.

2.2.- Capacitar a profesionales de los servicios públicos que integran los comités técnicos de evaluación del Sistema de Evaluación de Impacto Ambiental respecto a proyectos de inversión que puedan afectar al huemul y su hábitat.

2.3.- Involucrar a los legisladores regionales en el Plan de Acción.

2.4.- Fomentar la participación ciudadana.

2.5.- Considerar la recalificación de algunas de las áreas protegidas donde está presente el huemul (p.ej. de Reserva Nacional a Parque Nacional), considerando categorías que brinden mayor restricción al uso antrópico y/o cantidad de herramientas para la protección de la especie (CONAF).

2.6.- Tomar en cuenta el uso y relación de los Kawésqar con el huemul al diseñar las medidas de manejo de un ASPs, e incluir a la comunidad y sus representantes en la toma de decisiones.

3.- Enfermedades.

3.1.- Promover la realización de un análisis, utilizando un Sistema de Información Geográfica (SIG), para superponer zonas de sanidad animal con aquellas con presencia de huemul y/o hábitat apto para esta especie (se sugiere a SAG como organismo encargado).

3.2.- Solicitar, por parte de CONAF, el muestreo sanitario de huemules a SAG.

3.3.- Generar una sanción efectiva para la introducción ilegal de ganado a ASPs.
4.- Depredación y acoso por perros.

4.1.- Aplicar la modificación al Reglamento de la Ley de Caza que cambia la calificación de perros domésticos a "animal dañino" (en curso).

4.2.- Difundir y mejorar el SIG de perros asilvestrados, con énfasis en los hábitats del huemul.

4.3.- Promover la aplicación de ordenanzas municipales para el control de perros vagos en las comunas con presencia y hábitat de huemul (ver posibilidad de cooperación con el Sistema Nacional de Coordinación de Información Territorial, www.snit.cl).

5.- Caza furtiva.

5.1.- Promover la obtención de estatus de inspectores "Ad Honorem" para los guardaparques de CONAF (CONAF institución encargada).

5.2.- Concientizar al público sobre la importancia del huemul.

5.3.- Incorporar módulos educativos en los planes de educación ambiental de ASPs (CONAF) y de las escuelas vecinas a las ASPs. 5.4.- Solicitar a MBN que los administradores de áreas concesionadas con hábitat de huemul incorporen módulos educativos.

6.- Intervenciones transversales.

6.1.- Incluir en las bases del Plan de Acción la participación de las comunidades indígenas presentes en las zonas con presencia de huemul, tanto en la elaboración como implementación de medidas. Gestionar esto oficialmente con la Corporación Nacional de Desarrollo Indígena.

6.2.- Requerir que los proyectos de investigación en huemul que se desarrollen en la Zona Austral sean validados por el Grupo Zonal del Plan Nacional.

6.3.- Solicitar a las autoridades que los proyectos tanto públicos como privados que se desarrollen en hábitat con presencia histórica de huemul realicen Evaluaciones de Impacto Ambiental de alta rigurosidad científico-técnica y que incluyan tanto el área de impacto directo como indirecto. En el caso actual del Proyecto minero en Isla Riesco, solicitar que se incorporen medidas adecuadas para la protección de la totalidad de la población de 
huemul presente en el área (según los datos de presencia confirmada de la especie que se presentaron durante el taller para ese sector). Se sugiere que la Coordinadora Zonal envíe una copia del Plan de Acción y propuestas a la institución ambiental respectiva (COREMA Magallanes).

6.4.- Promover en la institucionalidad ambiental un mayor seguimiento de las resoluciones de calificación ambiental en el marco del SEIA.

6.5.- Promover la participación activa de los privados que realicen actividades económicas que se desarrollan en la distribución actual del huemul para que participen del Grupo Zonal
Austral (ganaderos, empresarios turísticos, etc.). 6.6.- Incorporar en las actuales Estrategias de Desarrollo Regional, Biodiversidad y Planificación Territorial (a nivel regional y comunal) la importancia de la conservación del huemul y su hábitat.

Análisis de Amenazas y Propuesta de Intervenciones para Manejo y Conservación de la Especie y su Hábitat:

Este grupo estuvo conformado por G. Acosta, J. C. Aravena, A. Bahamondes, F. Barrientos, H. Garde, A. Hinojosa, J. C. Marín, J. Paredes y A. Vila. Durante la discusión se identificaron las siguientes relaciones entre amenazas:

\begin{tabular}{|c|c|c|c|c|c|}
\hline Matriz de relación entre amenazas directas e indirectas & 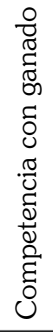 & 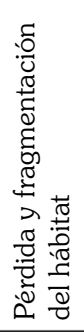 & 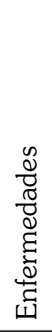 & 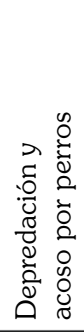 & 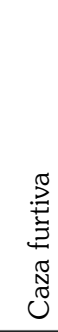 \\
\hline \multicolumn{6}{|l|}{ Falta de control efectivo en ASPs } \\
\hline \multicolumn{6}{|l|}{ Legislación sobre tenencia de perros inadecuada } \\
\hline \multicolumn{6}{|l|}{ Continuidad en las capacitaciones de personal de las ASPs } \\
\hline \multicolumn{6}{|l|}{ Necesidad de recursos humanos, financieros y materiales } \\
\hline \multicolumn{6}{|l|}{ Turismo no regulado en la distribución del huemul } \\
\hline \multicolumn{6}{|l|}{ Falta de coordinación dentro y entre servicios públicos y/u ONGs } \\
\hline Proyectos de inversión públicos/privados & & & & & \\
\hline
\end{tabular}

A continuación se enumeran las acciones propuestas por este grupo:

1.- Competencia con ganado.

1.1.- Determinar distribución y cantidad de ganado dentro de las ASPs.

1.2.- Eliminar el ganado en dichas áreas.

1.3.- Realizar una evaluación de áreas de alto riesgo de ingreso de ganado.

1.4.- Desarrollar un SIG superponiendo las áreas con presencia de ganado y de huemul.

1.5.- Realizar mesas de diálogo asociadas al Plan de Acción, con organismos como la Asociación Ganadera de Magallanes (ASOGAMA) y su equivalente en Aisén (OGANA), entre otros, para lograr su involucramiento en la conservación del huemul y el desarrollo de prácticas de manejo que favorezcan la recuperación de la especie.

2.- Pérdida y fragmentación del hábitat.

2.1.- Priorizar la fiscalización y cumplimiento de la normativa vigente en el hábitat con presencia de huemul.

2.2.- Realizar difusión y vigilancia para evitar incendios.

2.3.- Realizar un mapa de riesgo en SIG, superponiendo áreas con presencia de huemul y de proyectos de inversión/desarrollo.

3.- Enfermedades.

3.1.- Evaluar la necesidad de realizar tratamientos en huemules enfermos.

3.2.- Desarrollar un protocolo de acción frente a eventos de mortalidad de huemules asociados a enfermedades. 
3.3.- Desarrollar protocolo de toma y conservación de muestras.

3.4.- Desarrollar protocolo de necropsia.

3.5.- Formar un sub-grupo consultivo para asesorar en casos de brotes de enfermedades y los procedimientos a seguir.

3.6.- Capacitar a guardaparques sobre las enfermedades de animales domésticos que son transmisibles al huemul.

3.7.- Realizar análisis territorial de presencia de ciervo rojo y huemul.

4.- Depredación y acoso por perros.

4.1.- Prohibir la tenencia de mascotas en ASPs. 4.2.- Reforzar la prohibición del ingreso de perros y otras mascotas a las ASPs, concientizando a la comunidad sobre los inconvenientes que provocan en estas áreas.

4.3.- Trabajar en conjunto con las municipalidades para implementar un programa de esterilización y fomento de la tenencia responsable de mascotas en zonas de conflicto (por ejemplo, Pto. Edén y Cochrane).
4.4.- Fomentar la coordinación entre municipalidades, SAG y CONAF en el tema de tenencia responsable de perros y riesgos asociados.

4.5.- Buscar apoyo y financiamiento para el desarrollo de estas actividades.

5.- Caza furtiva.

5.1.- Aumentar el personal para efectivizar la fiscalización de la Ley de Caza, tanto fuera (SAG) como dentro (CONAF) de ASPs.

5.2.- Realizar un programa de educación ambiental para evitar la caza del huemul y lograr su valoración como especie heráldica.

Análisis de Amenazas y Propuesta de Intervenciones para Investigación y Monitoreo:

I. Avendaño, P. Cáceres, P. Corti, O. Guineo, C. Ivanovich, C. Moraga, M. Ruiz y R. Rodríguez formaron parte de este grupo de trabajo. El mismo identificó las siguientes relaciones entre amenazas. Asimismo, se estableció que algunas de las amenazas indirectas también se afectan entre sí: $\mathrm{d} \rightarrow \mathrm{b}, \mathrm{g}$; e $\rightarrow$ b, g (ver Tabla 1).

\begin{tabular}{|c|c|c|c|c|c|}
\hline Matriz de relación entre amenazas directas e indirectas & 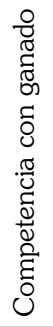 & 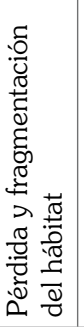 & 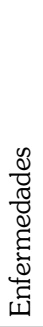 & 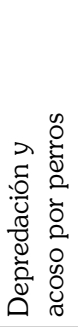 & 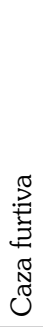 \\
\hline \multicolumn{6}{|l|}{ Continuidad en las capacitaciones de personal de las ASPs } \\
\hline \multicolumn{6}{|l|}{ Necesidad de recursos humanos, financieros y materiales } \\
\hline \multicolumn{6}{|l|}{ Turismo no regulado en la distribución del huemul } \\
\hline \multicolumn{6}{|l|}{ Falta de coordinación dentro y entre servicios públicos y/u ONGs } \\
\hline \multicolumn{6}{|l|}{ Monitoreos no estandarizados } \\
\hline \multicolumn{6}{|l|}{ Proyectos de inversión públicos/privados } \\
\hline Falta de investigación de la especie & & & & & \\
\hline
\end{tabular}

A continuación se enumeran las acciones propuestas por este grupo:

1.- Pérdida y fragmentación del hábitat.

1.1.- Determinación estado genético poblacional y flujo entre poblaciones.

2.- Falta de conocimiento y valoración de la especie.

2.1.- Determinar el nivel de conocimiento. 3.- Necesidad de recursos humanos, financieros y materiales:
3.1.- Solicitar una línea de financiamiento fiscal para realizar investigaciones sobre el huemul. 3.2.- Identificar otras posibles fuentes de financiamiento nacional $e$ internacional.

4.- Turismo no regulado.

4.1.- Desarrollar estudios conductuales en zonas con turismo.

4.2.- Establecer los presupuestos para realizar estos estudios, priorizando áreas con impacto turístico actual y futuro. 
5.- Falta de coordinación dentro y entre servicios públicos y ONGs.

5.1.- Incentivar alianzas estratégicas entre servicios públicos, organismos de investigación y ONGs.

6.- Monitoreo no estandarizado.

6.1.- Desarrollo de un taller teórico/práctico sobre monitoreo y evaluación de los protocolos utilizados.

6.2.- Generar una estandarización de métodos de captura.

7.- Proyectos de inversión públicos/privados.

7.1.- Determinar impactos realizando un monitoreo pre- y post-ejecución de proyectos.

8.- Falta de investigación sobre la especie.

8.1.- Incentivar la prospección de áreas aún no exploradas para obtener datos de distribución y abundancia en la zona.
8.2.- En áreas que cuentan con estudios sobre la especie (e.g. Fiordos Bernardo y Témpanos, Parque Nacional B. O'Higgins o el Parque Nacional T. del Paine), pasar a otra etapa de monitoreo de acuerdo a las necesidades de cada área.

8.3.- Cambiar y/o ajustar los protocolos de monitoreo en zonas ya estudiadas.

8.4.- Determinar dinámicas poblacionales, factores limitantes y estado sanitario.

8.5.- Incluir a las comunidades en las prospecciones de nuevos sitios.

Análisis de Amenazas y Propuesta de Intervenciones para Educación y Comunicación:

T. Gómez, C. Hernández, R. Ordóñez, G. Pérez, F. Repetto, D. Torres y N. Zambrano conformaron este grupo que identificó las siguientes relaciones entre amenazas.

\begin{tabular}{|c|c|c|c|c|c|}
\hline Matriz de relación entre amenazas directas e indirectas & 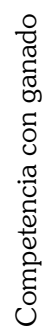 & 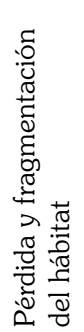 & 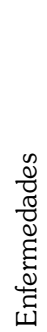 & 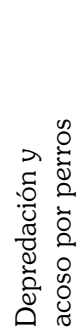 & 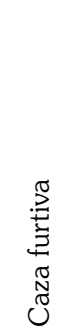 \\
\hline \multicolumn{6}{|l|}{ Falta de control efectivo en ASPs } \\
\hline \multicolumn{6}{|l|}{ Falta de conocimiento y valoración de la especie } \\
\hline \multicolumn{6}{|l|}{ Legislación sobre tenencia de perros inadecuada } \\
\hline \multicolumn{6}{|l|}{ Continuidad en las capacitaciones de personal de ASPs } \\
\hline \multicolumn{6}{|l|}{ Necesidad de recursos humanos, financieros y materiales } \\
\hline Proyectos de inversión públicos/privados & & & & & \\
\hline
\end{tabular}

A continuación se enumeran las acciones propuestas por este grupo:

1.- Depredación y acoso por perros.

1.1.- Fomentar la aplicación, por parte de las autoridades, de las ordenanzas municipales dirigidas a la tenencia responsable de mascotas, haciéndoles conocer su importancia en las zonas cercanas a poblaciones de huemul. 1.2.- Concientizar a los pobladores de zonas cercanas a poblaciones de huemul sobre la amenaza que significan los perros para los huemules, la importancia de esta especie y como ellos pueden colaborar a solucionar este problema.
2.- Falta de conocimiento y valoración de la especie. 2.1.- Diseñar e implementar una campaña de educación y comunicaciones tanto a nivel zonal como nacional.

2.2.- Realizar un diagnóstico sobre los problemas y públicos blanco prioritarios tanto a nivel zonal como nacional.

2.3.- Identificar a los distintos actores que podrían ayudar a crear una campaña de educación.

2.4.- Fomentar que los estudios de investigación sobre la especie contemplen el aspecto de difusión de la información a las personas directamente involucradas (p.ej. cazadores, 
estancieros), entregándoles además herramientas para mitigar la amenaza de la cual son partícipes.

2.5.- Identificar los distintos grupos prioritarios o blancos a quienes se les diseminará la información.

2.6.- Fomentar la educación y colaboración con cazadores, ya que ellos podrían ayudar a detectar irregularidades en las poblaciones de huemul (p.ej. enfermedades, detección presencia de especies introducidas como ciervos rojos).

3.- Falta de investigación sobre la especie.

3.1.- Iniciar un catastro de toda la información científica y no científica existente sobre el huemul.

3.2.- Crear un grupo de trabajo dentro del Plan Nacional para priorizar los vacíos de información y conocimiento.

Dentro del trabajo realizado por este grupo, se también identificó la necesidad y urgencia de diseñar e implementar una campaña de educación y comunicaciones, donde los potenciales grupos blanco deberían ser:

- Comunidades aledañas a poblaciones de huemul.

- Escuelas con certificación ambiental de comunidades aledañas a poblaciones de huemul.

- Profesores (educar a los educadores) de todo Chile.

- Guardaparques de ASPs ubicadas dentro de la distribución (actual e histórica) del huemul.

- Personal del SAG y autoridades municipales.

- Cazadores interesados en proteger/conservar nuestra fauna nativa.

- Turistas.

Se proponen además, las siguientes herramientas potenciales para utilizar en la difusión de información:

- Charlas, videos, documentales, folletos y talleres.

- Internet (facebook, listserve, blogs, web instituciones relacionadas CONAF, WCS, etc.).
- Campañas puerta a puerta.

- Difusión por radio y televisión.

- Difusión científica: Simplificar publicaciones y hacerlas accesibles en un lenguaje menos técnico (publicaciones amigables).

- Turismo social (escuelas y sociedad).

- Fomentar la realización de proyectos educativos (FPA y EXPLORA).

- Gestionar redes de apoyo, alianzas estratégicas y redes de monitoreo que incentiven el aviso de situaciones de interacción sociedad-huemul, como la caza furtiva.

- "Cicletada por el huemul" como la que se desarrolló hace años atrás.

- Mayor integración de las personas en los correos informativos sobre el huemul (p.ej. Boletín del Huemul).

- Creación de Unidades Pedagógicas en las escuelas de Chile.

- Integrar a currículum formal de educación en biodiversidad.

- Realizar Feria de Biodiversidad en Punta Arenas, integrándola con las ferias escolares y los grupos de Forjadores Ambientales.

\section{CONCLUSIONES}

Durante el Taller se hizo evidente la necesidad de establecer objetivos y, eventualmente, una meta, que sean específicos para la Zona Austral. Estos deben contemplar un componente cuantificable, con el fin de orientar las acciones $y$, al mismo tiempo, su monitoreo y evaluación. Es importante destacar que el Plan Nacional cuenta con un desglose de objetivos para los cuales se plantean actividades más específicas. Sin embargo, éstas no necesariamente son atingentes a la realidad de la Zona Austral o no son cuantificables en términos de evaluar el cumplimiento de la meta propuesta. Una forma de agilizar el establecimiento de objetivos zonales sería la formulación de enunciados preliminares a partir de los resultados de este taller, a ser desarrollados por el Coordinador y el Grupo de Trabajo Zonal, los cuales luego podrían ser circulados, discutidos y aprobados por los participantes del taller vía correo electrónico.

La identificación de cinco amenazas directas como prioritarias, así como las propuestas de medidas específicas para mitigarlas, son importantes avances para la elaboración de un Plan de Acción dirigido 
a mejorar la situación del huemul en la Zona. Las intervenciones propuestas variaron según el ámbito de discusión de cada grupo de trabajo. Sin embargo, algunas propuestas fueron recurrentes en más de un grupo. Entre ellas se puede destacar:

- Creación de un SIG que identifique las áreas con presencia de huemul y su vulnerabilidad frente a las amenazas (p.ej. presencia de baguales, centros urbanos y poblaciones rurales, proyectos de inversión, zonas de riesgo sanitario en términos de salud animal, etc.). Se estimó prioritario recopilar la información ya existente y estudiar la posibilidad de cooperación con proyectos relacionados (p.ej. SNIT, SAG y mapeo de distribución de huemul desarrollado por CODEFF, WCS y la Fundación Vida Silvestre Argentina).

- Coordinación entre instituciones para evitar que las amenazas detectadas ingresen a las ASPs o llevar a cabo acciones para erradicarlas cuando ya hayan ingresado. Esto involucra la promoción de la tenencia responsable de mascotas, las buenas prácticas de ganadería y silvicultura, la eliminación de animales baguales y la fiscalización de caza ilegal (algunos actores relevantes para la implementación serían las Municipalidades, SAG y CONAF).

- Difusión y concientización sobre la problemática del huemul a nivel de los tomadores de decisiones en áreas que estén relacionadas con las amenazas identificadas (servicios públicos encargados de analizar las EIAs, empresarios, cazadores, ganaderos, etc.). Se debe incluir información sobre medidas para evitar afectar a los huemules y su hábitat. En caso de que se requiera más información, el punto focal de contacto debe ser el Coordinador del Grupo Zonal. Esta difusión también debe complementarse con educación dirigida hacia otros actores (desde educación prebásica, básica y media, incluyendo zonas aledañas a poblaciones de huemul y en todo Chile).

La lista priorizada de amenazas directas que fue elaborada en este Taller debe ser tomada en cuenta en el proceso final de diseño del Plan de Acción, al señalar cuales son las intervenciones más apremiantes a desarrollar y, por lo tanto, que deberían recibir financiamiento en forma urgente. En el caso de las amenazas indirectas y sus intervenciones asociadas, la priorización de éstas surge naturalmente de su relación con las amenazas directas, si bien ésta varía dependiendo del ámbito de acción (Grupos de Trabajo) en las cuales se enmarcan. Se considera que estos ámbitos de acción son complementarios entre sí y, consecuentemente, no debería ser uno de ellos más importante que otro.

Debido a que la discusión debió extenderse por más de lo esperado, quedó pendiente una parte central del análisis propuesto, referida a los mecanismos de financiamiento y a la definición de que personas/instituciones estarán encargadas de implementar las actividades identificadas. Una vez que los mecanismos de financiamiento para las intervenciones sean establecidos, el último paso para la implementación del Plan de Acción es la definición de mecanismos para monitorear y evaluar, en base a indicadores cuantificables, el éxito de las medidas a desarrollar y, finalmente, el cumplimiento de los objetivos y la meta del Plan. Si bien los mecanismos específicos de monitoreo se definen en una última etapa, la necesidad de monitorear debe estar presente durante toda la elaboración del Plan, por ejemplo, al definir objetivos que sean específicos y mensurables. El monitoreo permite identificar claramente los resultados de la aplicación del Plan, establecer su nivel de efecto sobre el objeto de conservación y advertir de la necesidad de cambios en medidas que han mostrado no ser efectivas en el tiempo. Por otro lado, dada la baja representación de actores de la Región de Aisén, se considera necesario repetir esta experiencia de trabajo en dicha región y completar así la información necesaria para el desarrollo del Plan de la Zona Austral.

Finalmente, es importante hacer énfasis en que la situación crítica del huemul exige celeridad en la toma de decisiones y medidas concretas que apunten a revertir su declinación, aún a expensas de alcanzar los niveles de participación y/o la certeza científica idealmente necesarios para la toma de decisiones. Es decir, frente a la falta de información o participación se debe aplicar el principio precautorio y velar por evitar la extinción de esta especie heráldica y única del Cono Sur de Sudamérica. 


\section{AGRADECIMIENTOS}

Agradecemos el apoyo de la Universidad de Magallanes y el Instituto de la Patagonia por la gestión y facilitación de las dependencias del Centro Austral Antártico para el desarrollo de este Taller, en particular al Dr. Carlos Ríos, como así también la invitación a publicar estas Actas en los Anales del Instituto de la Patagonia. Asimismo, queremos destacar el apoyo financiero de Michel Durand (CAZ), la Weeden Foundation, WCS, CEQUA y CONAF para organizar este Taller. Finalmente, extendemos nuestro agradecimiento a todos los participantes del Taller, que a través de su activa y comprometida participación hicieron posible el comienzo del proceso para desarrollar un Plan de Acción para la Conservación del Huemul en la Zona Austral de Chile.

\section{LITERATURA CITADA}

Caughley, G. 1994. Directions in Conservation Biology. Journal of Animal Ecology 63:215-244.

CONAF \& CODEFF 2001. Plan para la conservación del huemul del sur, Hippocamelus bisulcus, en Chile. Santiago, Chile. 40 pp.

CONAF, SAG \& CONAMA 2009. Plan nacional de conservación del huemul (Hippocamelus bisulcus, Molina 1782) en Chile. 2008 - 2012. Taller participativo para la elaboración del plan, Puerto Fuy, Agosto de 2007. Santiago, Chile. 50 pp.

Diamond, J. 1984. Historic extinctions: A rosetta stone for understanding prehistoric extinctions. In: Martin, P.S. \& F. Klein (eds.): Quarternary extintions: a prehistoric revolution. pp. 73-97. University of Arizona Press, Tucson.

Drouilly, P. 1983. Recopilación de antecedentes biológicos y ecológicos del huemul chileno y consideraciones sobre su manejo. Boletín Técnico CONAF 5:1-57.

Jiménez, J., G. Guineo, P. Corti, J.A. Smith, W. Flueck, A.R. Vila, Z. Gizejewski, R. Gill, B. McShea \& V. Geist 2008. Hippocamelus bisulcus. In: IUCN 2010. IUCN Red List of Threatened Species. Version 2010. 4. <www. iucnredlist.org $>$.

López, R., A. Serret, R. Faúndez \& G. Palé 1998. Estado del conocimiento actual de la distribución del huemul (Hippocamelus bisulcus, Cervidae) en Argentina y Chile. FVSA/WWF/ CODEFF. Concepción, Chile. 32 pp.

Redford, K.H. \& J.F. Eisenberg 1992. Mammals of the Neotropics. Volume 2. The Southern Cone: Chile, Argentina, Uruguay, Paraguay. University of Chicago Press, Chicago, USA. $624 \mathrm{pp}$.

Vila, A.R., R. López, H. Pastore, R. Faúndez \& A. Serret 2006. Current distribution and conservation of the huemul (Hippocamelus bisulcus) in Argentina and Chile. Mastozoología Neotropical 13:263-260.

Vila, A.R., C.E. Saucedo, D. Aldridge, E. Ramilo \& P. Corti 2010. South Andean Huemul (Hippocamelus bisulcus, Molina 1782). In: Barbanti Duarte, J.M. \& S. González (eds.). Neotropical cervidology: Biology and medicine of Latin American deer, pp. 89-100. FUNEP-IUCN, SP, Brazil.

WCS. 2004. Valoración espacial de actividades humanas. Programa Paisajes Vivientes. Manual Técnico 1. WCS, NY, USA. 12 pp.

WCS. 2006. Building biological and threats landscapes from ecological first principles, a step-by-step approach. Living Landscape Program. Technical Manual 6. WCS, NY, USA. 20 pp. 
ANEXO 1

Listado de asistentes al Taller.

\begin{tabular}{|c|c|c|}
\hline $\mathbf{n}^{\circ}$ & Nombre & Institución \\
\hline 1 & Gerardo Acosta & $\mathrm{UACH}$ \\
\hline 2 & Paola Acuña & CEQUA \\
\hline 3 & Chelsea Ams & NOMADAS \\
\hline 4 & Juan Carlos Aravena & CEQUA \\
\hline 5 & Iván Avendaño & CONAF \\
\hline 6 & Andrea Bahamondes & Dpto. ASPs CONAF- Región de Aisén \\
\hline 7 & Fredy Barrientos & CONAF \\
\hline 8 & José Luis Cabello & SAG \\
\hline 9 & Patricio Cáceres & Hostería San Isidro \\
\hline 10 & Rafael Contreras & CONAF \\
\hline 11 & Paulo Corti & UACH - U. Bio-Bío \\
\hline 12 & Cristofer de la Rivera & Instituto Isla Negra \\
\hline 13 & Daniela Droguett & WCS \\
\hline 14 & Claudia Espinoza & Periodista \\
\hline 15 & Elena Garde & Veterinarios sin Fronteras - Canadá \\
\hline 16 & Teresita Gómez & CORMUPA \\
\hline 17 & Oscar Guineo & Investigador independiente \\
\hline 18 & Carla Henríquez & CEQUA \\
\hline 19 & Juan Marcos Henríquez & UMAG \\
\hline 20 & Carla Hernández & CONAF \\
\hline 21 & Ana Hinojosa & CONAF - Chillan \\
\hline 22 & Cristóbal Ivanovich & CONAF \\
\hline 23 & Rodrigo López & CODEFF - AUREN \\
\hline 24 & Juan Carlos Marín & Univ. del Bio-Bío \\
\hline 25 & Ann Meidinger & NOMADAS \\
\hline 26 & Claudio Moraga & WCS \\
\hline 27 & Ricardo Ordóñez & Escuela G-4 Puerto Natales \\
\hline 28 & José Paredes & CONAF \\
\hline 29 & Rodrigo Pérez & CEQUA \\
\hline 30 & Guillermo Pérez & Veterinarios sin Fronteras - Canadá \\
\hline 31 & Fiorella Repetto & WCS \\
\hline 32 & Carlos Ríos & UMAG \\
\hline 33 & Rodrigo Rodríguez & CONAF \\
\hline 34 & Mauricio Ruiz & CONAF \\
\hline 35 & Guillermo Santana & CONAF \\
\hline 36 & Carlos Silva & Instituto Isla Negra \\
\hline 37 & Nicolás Soto & SAG \\
\hline 38 & Juan Sotomayor & Independiente \\
\hline 39 & Gregor Stipicic & Fundación Yendegaia \\
\hline 40 & Juan Carlos Tonko & Comunidad Kawésqar \\
\hline 41 & Juliana Torres & CEQUA \\
\hline 42 & David Torres & NOMADAS \\
\hline 43 & Claudio Venegas & Instituto de la Patagonia \\
\hline 44 & Alejandro Vila & WCS \\
\hline 45 & Neftaly Zambrano & CONAF \\
\hline 46 & Alejandra Zúñiga & CONAF \\
\hline
\end{tabular}


ANEXO 2

Listado de las exposiciones presentadas durante el Taller "Hacia un Plan de Acción para la Conservación del Huemul en la Zona Austral".

- El Plan Nacional y la conformación del Grupo Zonal Austral (Ana Hinojosa, CONAF).

- La distribución del huemul a escala global con énfasis en la Zona Austral (Rodrigo López, CODEFF y AUMEN).

- La visión del huemul desde la perspectiva de los pueblos originarios (Juan Carlos Tonko, Comunidad Kawésqar).

- Genética y evolución de las poblaciones de huemul (Juan Carlos Marín, Universidad del Bío-Bío).

- El huemul en áreas protegidas y no protegidas: Esfuerzos de conservación en el Parque Nacional Torres del Paine y en la Península de Brunswick (Gladys Garay y Oscar Guineo, Independientes, y Fredy Barrientos, CONAF).

- Esfuerzos de conservación en los Fiordos Témpanos y Bernardo, Parque Nacional Bernardo O'Higgins (José Paredes e Iván Avendaño, CONAF, Alejandro Vila, Fiorella Repetto y Daniela Droguett, WCS).

- El huemul en las áreas protegidas: Aspectos sanitarios relacionados con la población de los Fiordos Témpanos y Bernardo (Ana Hinojosa, José Paredes e Iván Avendaño, CONAF).

- Estado de situación y avances en el conocimiento del huemul en Aisén (Paulo Corti, UACh). 


\section{ANEXO 3}

Resúmenes de algunas de las presentaciones orales del Taller.

DISTRIBUCIÓN DEL HUEMUL (Hippocamelus bisulcus)

EN LA ZONA AUSTRAL DE CHILE.

Rodrigo López Rübke ${ }^{1}$

El huemul, especie emblemática de Chile y clasificada en Peligro Crítico de Extinción por la UICN, tenía una distribución original que se extendía en forma continua desde el río Cachapoal en la región del Libertador B. O'Higgins hasta la península de Brunswick en el estrecho de Magallanes. Actualmente, se ha sugerido una reducción de su distribución en el territorio nacional superior al 50\%, habiéndose extinguido en las regiones del Libertador B. O'Higgins, el Maule y la Araucanía; mientras que no existirían registros actuales sobre su presencia entre los $38^{\circ}$ y los $41^{\circ}$ L.S.

Los estudios relacionados con el conocimiento de la distribución actual de la especie en el país han sido desarrollados a través de encuestas y talleres con expertos, publicaciones científicas, entrevistas con guardaparques y revisión de informes técnicos de distintas iniciativas realizadas en terreno por diversas organizaciones, como la Corporación Nacional Forestal, el Ministerio de Bienes Nacionales, el Comité Nacional Pro Defensa de la Fauna y Flora, la Universidad de Concepción, la Universidad Austral de Chile, la Universidad del Bio-Bío, AUMEN ONG, Fundación CEQUA, Wildlife Conservation Society e investigadores privados.

En la zona austral, que comprende desde la cuenca del río Cisnes, en la región de Aisén, hasta el Estrecho de Magallanes, en la región del mismo nombre, se han registrado 200 puntos o sitios públicos y privados con presencia efectiva de la especie. Por esta razón, se sugiere que este territorio concentra las mayores poblaciones de huemul en Chile. Un gran porcentaje de estos puntos de presencia se ubican en áreas protegidas, como los Parques Nacionales Laguna San Rafael, Torres del Paine y Bernardo O'Higgins; las Reservas Nacionales Lago Carlota, Lago Las Torres, Mañihuales, Río Simpson, Cerro Castillo, Lago Cochrane, Jeinimeni, Katalalixar, Alacalufes y Laguna Parrillar; y los Bienes Nacionales Protegidos como Laguna Caiquenes, El Mosco, Santa Lucia, Laguna Vera, Ventisquero Montt, Batchelor y Cabo Froward.

En la actualidad, las poblaciones de huemul localizadas en la zona austral son las que presentan el menor grado de fragmentación y sus hábitats son continuos, ya presenta baja densidad de población humana, aislamiento geográfico y una importante red de Áreas Silvestres Protegidas Públicas y Privadas, que son continuas, de gran tamaño y les brindan protección.

\section{BUSCANDO GENES EN EL HUEMUL.}

Juan Carlos Marín ${ }^{2}$

El origen y formación de los diferentes linajes que han llegado a conformar las especies actuales de cérvidos Neotropicales, probablemente, ocurrió en el Pleistoceno. La aparición del género Hippocamelus y el consiguiente proceso de diferenciación al interior de este, pudo ocurrir a medida que las poblaciones del linaje ancestral se extendieron hacia el sur, a través de los Andes, llegando a originar las dos especies actuales del género: la taruca y el huemul. Con el uso de marcadores del mtDNA, aquí analizamos la diversidad genética y estructura filogeográfica de 20 poblaciones de huemul de Argentina y Chile.

Nuestros resultados muestran que las poblaciones de huemul presentan un alto grado de diversidad genética materna y signos de expansión poblacional reciente. El diagrama del árbol de expansión mínima

\footnotetext{
Proyecto Huemul, Comité Nacional Pro Defensa de la Fauna y Flora \& Frankfurt Zoological Society \& AUMEN ONG.
} Universidad del Bio-Bío, Proyecto DIUBB 082409 1/R, FONDECYT Iniciación 11080098 
de los haplotipos de la región control de huemul, muestra ausencia de formas genéticas diferenciadas que permitan afirmar la existencia de subespecies. Sin embargo, el análisis de la asignación de individuos a grupos de poblaciones, considerando la información geográfica y genética, demuestra la presencia de tres grupos que evidencian una historia fuertemente marcada por las oscilaciones climáticas durante el Cuaternario. La información mitocondrial permite proponer la existencia de refugios glaciares, ubicados al este y al oeste de la distribución de los hielos, durante el Último Máximo Glacial. Es posible que eventos paleoclimáticos como el Último Máximo Glacial, habrían producido reducciones drásticas del tamaño poblacional o "cuellos de botella" que se reflejan en el patrón de diversidad genética actual de las poblaciones y la distribución de desigualdades.

El siguiente paso de este estudio será apunta a la optimización de un set de tres marcadores moleculares independientes (AMELX-Y, ZFX-Y y Sry) que permiten identificar el sexo de una muestra, así como la optimización de marcadores micro-satélite específicos para la especie.

\section{SOCIOECOLOGÍA DE LA POBLACIÓN DE HUEMULES DEL PARQUE NACIONAL TORRES DEL PAINE 1999-2009.}

Gladys Garay \& Oscar Guineo 3

El huemul (Hippocamelus bisulcus) es una especie de ciervo que habita sólo en Chile y Argentina, en la zona cordillerana que ambos países comparten, concentrándose en la zona sur y austral. En particular, este estudio se refiere a una población que se encuentra en el Parque Nacional Torres del Paine, en la Región de Magallanes, Chile. Entre los años 1999 y 2009 estudiamos la población de huemules presente en el sector lago Grey, en el extremo oeste del Parque, abordando diversos aspectos de la socioecología del huemul. Se comenzó con una prospección inicial para estimar el tamaño de la población en dicha área, la cual fue estimada en a lo menos 60 animales. Luego se determinó la estructura social y el uso de hábitat según la distribución y el movimiento de los grupos cercanos a la Guardería Grey, obteniendo una estructura social basada en la pareja macho-hembra y la identificación de 6 categorías de grupos diferentes. A partir de las observaciones de uso de hábitat y desplazamientos, se registró un rango de hogar promedio anual de 309,9 ha. En cuanto a la reproducción, el celo ocurre en otoño (marzo-abril) y las pariciones a mediados de la primavera (octubre-noviembre). La actividad diaria incluyó la alimentación (aproximadamente el 50\% del tiempo), descanso, rumia, desplazamientos caminando, vigilancia, acicalamiento, juego y conductas sociales asociadas. En este estudio se realizó la primera marcación de cervatillos de huemul en Chile, lográndose 16 animales marcados con aretes numerados. El uso de esta metodología permitió obtener una mayor información sobre esta población, incluyendo datos de longevidad de a lo menos 7 años hasta la fecha de este reporte.

\section{ESFUERZOS DE CONSERVACIÓN EN LOS FIORDOS TÉMPANOS Y BERNARDO, PARQUE NACIONAL BERNARDO O'HIGGINS (PNBO).}

\section{Alejandro Vila ${ }^{4}$, José Paredes ${ }^{5}$, Iván Avendaño ${ }^{4}$, Daniela Droguett ${ }^{3} \&$ Fiorella Repetto $^{3}$}

Los estudios realizados con huemules en el PNBO se remontan a la década del 90, cuando Alejandro Frid evaluó el uso del hábitat y la organización social de este cérvido nativo. Dichos estudios se realizaron antes de que se introdujera ganado vacuno en el valle Huemules, lo cual le permitió a este autor

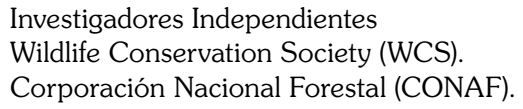


comparar la respuesta del huemul frente a la presencia de vacunos. Esta introducción habría provocado nuevas amenazas directas para esta población, como competencia, riesgo de transmisión de enfermedades e incremento de la presencia humana, asociada con episodios de caza y/o persecución de perros.

En el año 2002, CONAF y el Centro de Aclimatación Zoológica La Dehesa (CAZ) firmaron un convenio de cooperación público-privada para mitigar estas amenazas y proteger las poblaciones con mayor densidad de huemules conocidas. WCS participó del mismo brindando asesoramiento técnicocientífico y aportando financiamiento para el desarrollo de algunas actividades específicas. Este convenio se mantuvo activo hasta el 2005. Sin embargo, WCS siguió apoyando esta iniciativa. Finalmente, en el 2007, CONAF firmó un nuevo convenio con WCS para promocionar y fomentar la conservación del patrimonio natural de Chile, con énfasis en la XII Región. Entre sus objetivos se promociona y fomenta la protección del huemul en el PNBO, a través de estimular el desarrollo de investigaciones sobre la especie y la biodiversidad de los ecosistemas australes. A partir del 2008 se comenzaron a evaluar alternativas para implementar este último convenio.

Durante el año 2004 se realizó un ejercicio de planificación estratégica para identificar amenazas y acciones necesarias a implementar en el área. De esta forma fue posible identificar amenazas indirectas asociadas con competencia y enfermedades por introducción de ganado, incremento de caza ilegal y depredación/acoso por perros, como la situación económica de las comunidades vinculadas con el PNBO; su percepción sobre la situación del huemul; la falta de conocimiento, control y vigilancia, recursos humanos y financieros; y la necesidad de contar con instancias de capacitación e información. Por esta razón, las acciones planificadas se enfocaron en mitigar o eliminar estas amenazas. Dentro de este marco, CAZ y WCS han aportado un mínimo de 600 mil dólares para la construcción y equipamiento de la Estación Fiordo Témpanos $\left(170 \mathrm{~m}^{2}\right)$ y el Refugio Fiordo Bernardo $\left(60 \mathrm{~m}^{2}\right)$, además de la embarcación Jekcal. En un accionar conjunto con CONAF se contrataron guardaparques, se los equipó y capacitó en técnicas de monitoreo y se diseñó e implementó un programa básico de monitoreo que fue implementado por los guardaparques. También se realizaron campañas de control de ganado y estudios de abundancia, uso espacial, patrones de actividad y ecología alimenticia de huemul. Los resultados obtenidos tras las acciones educativas realizadas han sido variados, como talleres con las escuelas locales y publicaciones educativas, enmarcados en proyectos FPA y Explora-CONICYT.

\section{ASPECTOS SANITARIOS DEL HUEMUL EN TRES SECTORES DEL PARQUE NACIONAL BERNARDO O'HIGGINS (PNBO).}

\section{A. Hinojosa ${ }^{6}$, J. Paredes ${ }^{5}$, I. Avendaño ${ }^{5}$, A. Silva ${ }^{5}$, M. Quezada $^{7}$, C. Brevis ${ }^{6}$ \& D. Gonzalez-Acuña ${ }^{6}$}

Desde el año 2005 se han observado huemules con claudicaciones y con diferentes grados de lesiones en sus extremidades en tres valles del PNBO. Las causas que originan estas cojeras podrían ser variadas, entre las cuales se pueden mencionar, disputas entre machos durante el período de reproducción, traumatismos post cruza, aspectos ambientales, topográficos y sanitarios, entre otros. Algunos de estos factores podrían ser los que operan como puerta de entrada para agentes infecciosos secundarios que complican, posteriormente, la lesión. La importancia de este cuadro, radica en que los huemules afectados quedan a merced de su depredador nativo en la zona, el zorro culpeo (Lycalopex culpaeus Molina, 1782).

Para determinar el origen de esta problemática se está realizando un trabajo de seguimiento de los animales con claudicaciones, toma de muestras en ejemplares afectados y análisis en laboratorio, así como la colecta de fecas de zorro para analizar el grado de interacción con las poblaciones de huemules locales. Dicho trabajo es realizado conjuntamente por profesionales de CONAF y la Universidad de Concepción.

Departamento de Patrimonio Silvestre, Corporación Nacional Forestal.

Facultad de Medicina Veterinaria, Universidad de Concepción, sede Chillán. 